\title{
Fluctuations of orientational order and clustering in a two-dimensional colloidal system under quenched disorder
}

\author{
Tobias Horn, ${ }^{1, *}$ Sven Deutschländer, ${ }^{2}$ Hartmut Löwen, ${ }^{1}$ Georg Maret, ${ }^{2}$ and Peter Keim ${ }^{2}$ \\ ${ }^{1}$ Institut für Theoretische Physik II, Heinrich-Heine-Universität Düsseldorf, D-40225 Düsseldorf, Germany \\ ${ }^{2}$ Fachbereich für Physik, Universität Konstanz, D-78464 Konstanz, Germany
}

(Received 10 September 2013; published 11 December 2013)

\begin{abstract}
Using both video microscopy of superparamagnetic colloidal particles confined in two dimensions and corresponding computer simulations of repulsive parallel dipoles, we study the formation of fluctuating orientational clusters and topological defects in the context of the KTHNY-like melting scenario under quenched disorder. We analyze cluster densities, average cluster sizes, and the population of noncluster particles, as well as the development of defects, as a function of the system temperature and disorder strength. In addition, the probability distribution of clustering and orientational order is presented. We find that the well-known disorder-induced widening of the hexatic phase can be traced back to the distinct development characteristics of clusters and defects along the melting transitions from the solid phase to the hexatic phase to the isotropic fluid.
\end{abstract}

DOI: 10.1103/PhysRevE.88.062305

PACS number(s): 82.70.Dd, 64.70.D-, 61.20.Ja, 64.70.pv

\section{INTRODUCTION}

The controversy about the microscopic process controlling melting in two dimensions has lasted for several decades. According to the widely accepted theory by Kosterlitz, Thouless, Halperin, Nelson, and Young (KTHNY) [1-5], two-dimensional (2D) melting is a sequence of two continuous phase transitions. The solid and isotropic fluid phases are separated by an intermediate anisotropic fluid phase with quasi-long-range sixfold orientational order, called the hexatic phase. The KTHNY scenario further suggests that the two continuous transitions are driven by the dissociation of thermally activated topological defects [2,5]. Given the sixfold symmetry of a triangular lattice, a dislocation constitutes a topological defect which consists of a pair of particles with five and seven nearest neighbors, respectively. Following the predictions of the KTHNY theory, dislocations are bound into neutral pairs in the solid phase, and orientational order is long-range; i.e., the orientational correlation function attains a finite value. As pointed out by Mermin [6], the translational order of the crystalline state is only quasi-long-range due to long-wavelength fluctuations. Mediated by the unbinding of dislocation pairs into isolated dislocations, the transition to the hexatic phase takes place at a temperature $T_{m}$ [4]. Although translational order is destroyed by the presence of isolated dislocations, orientational order persists in the hexatic phase on a quasi-long-range scale [3,4]. Thus, in the hexatic phase, the orientational correlation function decays algebraically as a function of the separation distance. The transition from the hexatic to the isotropic fluid phase at the temperature $T_{i}>T_{m}$ is marked by the unbinding of dislocations into single five- and sevenfold point defects, which are referred to as disclinations. In the isotropic fluid, orientational order vanishes and the orientational correlation function decays exponentially.

Alternative approaches to melting in two dimensions have been conceived where the melting process is modeled as a single first-order transition $[7,8]$. However, throughout numerous experimental and simulation studies, the KTHNY

*thorn@thphy.uni-duesseldorf.de theory could not be consistently verified or refuted [9]. Instead, evidence was found that the melting process strongly depends on the pair interaction of the system at hand [10]. While systems governed by very short-range or hard-core interactions are reported to exhibit coexisting phases [11-13], thus contradicting the notion of a continuous transition, reports on soft repulsive particles in two dimensions favor the KTHNY scenario [14-16]. For particles interacting via long-range dipolar interactions scaling with the inverse cube of the particle separation, the KTHNY scenario has been unambiguously confirmed [17-19]. Video-microscopy experiments on superparamagnetic colloidal particles pending at an air-water interface, where an external magnetic field induces dipolar moments perpendicular to the surface, have verified the predictions of the KTHNY scenario in detail [20,21], including the elastic properties related to the mechanism of defect unbinding [22]. Furthermore, this setup was studied after a quench by instantaneously increasing the external magnetic field [23-25]. Thereby, crystallization occurred without any evidence of the hexatic phase. Instead, the local formation of crystallites, which gradually merged into larger crystalline patches, leads to a polycrystalline state [23].

According to theoretical predictions by Nelson and coworkers, the two-stage melting scenario persists in the presence of weak disorder [26,27], and the stability range of the hexatic phase widens with increasing disorder. While $T_{i}$ is predicted to be largely unaffected by disorder, $T_{m}$ decreases with increasing disorder until, eventually, no crystalline state can be established [26]. While the original work suggested a reentrant melting at low temperatures in the presence of disorder [26], later works revised this idea and a final prediction of the stability range of the ordered (i.e., solid) phase in the temperature-disorder plane was stated in [28]. Further numerical studies confirmed the topography of the ordereddisordered phase diagram [29], albeit the hexatic phase was not resolved. Experimental realizations of colloidal systems under quenched disorder were explored, where quenched disorder was embodied by larger particles dispersed in an array of smaller particles. Thereby, the solid phase exhibited the properties of an hexatic glass [30]. Static and dynamic properties of the crystalline state in the presence of a random pinning 
potential were studied in [31]. Additionally, the properties of a disorder-induced glass transition have been thoroughly accounted for [32-34]. Recent studies on experimental model systems like magnetized monolayers [35] or single-layer complex plasmas [36] have increased our understanding of the $2 \mathrm{D}$ crystallization process.

In a previous work [37], we proposed an experiment on superparamagnetic colloidal particles on a substrate, where quenched disorder is realized by randomly pinning particles to the substrate. Probing different regions of the sample with varying fractions of pinned particles, the melting process was analyzed for various degrees of disorder. Thus, the specific dependency of the transition temperatures $T_{i}$ and $T_{m}$ on disorder predicted in [26] and [27] was verified and a broadening of the hexatic phase could be observed. Furthermore, computer simulations on parallel dipoles in two dimensions were conducted, which exhibited very good agreement with the experimental data.

In this paper, we extend our previous findings on the disorder-induced melting process [37] with a detailed analysis of orientational clusters which show strong fluctuations in time. The development of these clusters is discussed globally and locally as a function of the induced disorder in the form of particle pinning. Dealing with fluctuations on various length scales in continuous 2D melting, this is an essential part of understanding the conservation of the continuous nature of the transitions in the presence of weak induced disorder. In the isotropic liquid phase, the emergence of small clusters corresponds to strongly fluctuating patches of high orientational order, which gradually merge into bigger clusters at lower temperatures. The emergence of a single cluster spanning the entire system occurs close to the isotropic $\rightarrow$ hexatic transition. As discussed in [37], the orientational order parameter exhibits spatiotemporal critical(-like) fluctuations close to the isotropic-hexatic transition and throughout the hexatic phase. In the coarsened picture of orientational clusters, these fluctuations correspond to regions excluded from clusters. Albeit the description of orientational clusters is formally similar to the analysis of heterogeneous crystallites in a quenched colloidal system as explored in [23], orientational clusters are not invoked by a quench of the system but rather exhibit the nature of the continuous transition. As we observe in computer simulations and experiment, the number and size of these clusters display characteristic properties in the isotropic fluid, hexatic fluid, and solid phase. Analyzing setups with varying degrees of quenched disorder, the broadening of the hexatic phase can be traced back to inhibited cluster formation. As indicated by a comparison of our results to bulk reference simulations, the formation of orientational clusters is not induced by the presence of pinned particles but can be observed in a pure system as well. The analysis of the density of topological defects indicates that the widened stability range of the hexatic phase reported in [37] is in fact associated with an increased abundance of isolated dislocations. This is consistent with the KTHNY scenario, which predicts that isolated dislocations emerge in the hexatic phase and that quenched disorder triggers the unbinding of dislocation pairs. Additionally, we present further analysis of our experimental and numerical data supporting the continuous nature of the isotropic-hexatic phase transition.
The paper is organized as follows: In Sec. II, we describe the experiment. The simulation technique is reported in Sec. III. Section IV contains a description of our methods and introduces the definition of clusters. Our analysis of cluster formation and the finite-size behavior of global orientational order is given in Sec. V. Topological defects are analyzed in Sec. VI. Finally, Sec. VII provides the conclusion.

\section{EXPERIMENTAL SETUP}

We study a suspension of superparamagnetic colloidal particles in two dimensions. Confined within a cylindrical glass cell of $5-\mathrm{mm}$ diameter, the particles sediment due to gravity and form a monolayer on the bottom glass plate. Quenched disorder is imposed by the pinning of a small amount of particles which are attached to the glass substrate due to van der Waals interactions and chemical reactions. The particles have a diameter of $d=4.5 \mu \mathrm{m}$ and a mass density of $1.7 \mathrm{~kg} / \mathrm{dm}^{3}$. The short-time lateral diffusion constant on the glass substrate is $D=0.0295 \mu \mathrm{m}^{2} / \mathrm{s}$ and the Brownian time scale corresponds to $\tau_{B}=(d / 2)^{2} / D \approx 170 \mathrm{~s}$. Although thermal tearing or the creation of new pinning connections does alter the distribution of obstacles, the pinned particles remain fixed on the time scale of our measurements $\left(\approx 60 \tau_{B}\right)$. Applying an external magnetic field $H$ perpendicular to the substrate plane, dipole moments are induced. Due to the parallel alignment of dipoles, the pair interaction scales with the inverse cube of the particle separation. The pair interaction strength can be readily expressed by the dimensionless parameter [38]

$$
\Gamma=\frac{\mu_{0}(\chi H)^{2}}{4 \pi a^{3} k_{B} T},
$$

where $a$ is the mean particle distance, $\chi$ denotes the magnetic susceptibility, and $k_{B} T$ is the thermal energy. The definition $a=(\pi n)^{-1 / 2}$ allows us to calculate the dipolar distance dependence of the pair potential from the 2D particle number density $n$ (including a geometrical prefactor). By changing the magnetic field, the system can be effectively "heated" or "cooled" homogeneously. We study the melting process in three sample regions with varying pinning fractions, ranging from approx. $0.5 \%$ to $0.8 \%$. While the entire system comprises $>10^{5}$ particles, each monitored region contains $\approx 5 \times 10^{3}$ particles. The colloidal ensemble is melted from an equilibrated crystalline state by decreasing $H$ in small steps. After each step, the system is allowed to equilibrate for at least $24 \mathrm{~h}$ before particle trajectories are recorded for $2.7 \mathrm{~h}$ via video microscopy [39].

\section{SIMULATION}

Additionally, we perform computer simulations of pointlike superparamagnetic particles in two dimensions which interact via the purely repulsive pair potential of parallel dipoles. Standard metropolis Monte Carlo (MC) simulations were conducted on the NAT ensemble, with $A$ denoting the area of the square simulation cell. The particle number was fixed at $N=16000$ and periodic boundary conditions were applied. Particle interactions are calculated via the truncated 
and shifted potential,

$$
U\left(r_{i j}\right)= \begin{cases}\Gamma\left(\left(r_{i j} / a\right)^{-3}-\left(r_{c} / a\right)^{-3}\right), & r_{i j}<r_{c}, \\ 0, & r_{i j} \geqslant r_{c},\end{cases}
$$

where the cutoff radius $r_{c} / a=10$ is chosen. The initial state of the simulation is obtained by successively attempting to place particles at random positions within the simulation cell, while the minimum distance between particles is restricted to $r_{i j} \geqslant a$. The resulting configurations corresponds to an isotropic liquid of hard disks with a packing fraction of $0.25 \%$. Quenched disorder is introduced by pinning a randomly chosen subset of particles to their initial position and rendering them immobile for the entire simulation run. Within statistical accuracy, this procedure models the distribution of pinning sites observed in the experiment. Each particular pinning fraction is sampled with at least 15 statistically independent distributions of pinned particles. Although typically MC methods are employed to study static properties in equilibrium states, the underlying phase-space sampling provides a suitable means for studying dynamic processes too [40]. To estimate the Brownian time scale in terms of MC sweeps (i.e., attempted moves per particle), the diffusion of particles is observed in a dilute bulk suspension. Thus, we find that the experimental Brownian time $\tau_{B}$ corresponds to $\approx 36 \mathrm{MC}$ sweeps. Starting in the isotropic fluid phase $\left(\Gamma^{-1}=0.0167\right)$, a full freezing and melting cycle is conducted for each pinning configuration. Note that during this process, the particular distribution of pinned particles remains fixed. At each step of $\Gamma$, the system is equilibrated for up to $5 \times 10^{5} \mathrm{MC}$ sweeps $\left(\approx 13800 \tau_{B}\right)$, after which data are acquired over $10^{5} \mathrm{MC}$ sweeps. For each pinning fraction explored, the observables obtained at a given value of $\Gamma$ are averaged over all sample realizations of disorder. As a reference, the same freezing and melting cycle is conducted for a pure bulk system, where all particles are mobile.

\section{METHODS}

Sixfold orientational order can be expressed in terms of the bond order parameter

$$
\psi_{6, j}=\frac{1}{n_{j}} \sum_{k=1}^{n_{j}} e^{i 6 \theta_{j k}}
$$

where the sum goes over all $n_{j}$ nearest neighbors of particle $j$, and $\theta_{j k}$ is the angle of the $k$ th bond with respect to a certain reference axis. For a particle embedded in a perfect hexagonal crystal at zero temperature, the magnitude of $\psi_{6}$ is 1. It decreases as the neighbor particles deviate from their ideal lattice positions, e.g., almost vanishes for a five- or sevenfold coordinated defect. Here, nearest neighbors of a particle are determined via the Voronoi construction. In order to analyze orientational clusters and fluctuations of the order parameter, we consider the local time-averaged director $\Psi_{6, i}$,

$$
\Psi_{6, i}(t)=\frac{1}{\Delta_{t}} \int_{t-\Delta_{t}}^{t} \psi_{6, i}\left(t^{\prime}\right) d t^{\prime} .
$$

For both experiment and simulation, the averages are conducted over a time frame $\Delta_{t} / \tau_{B} \geqslant 50$. Furthermore, the global
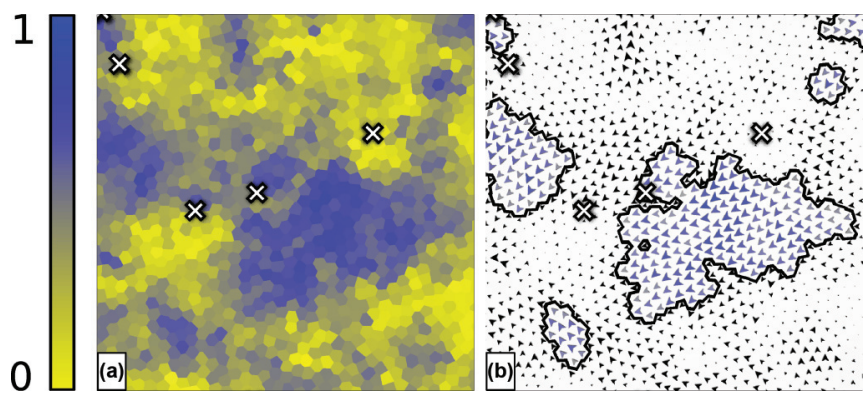

FIG. 1. (Color online) Simulation snapshots illustrating orientational clusters close to the fluid $\rightarrow$ hexatic transition $\left(\Gamma^{-1}=\right.$ $0.0149)$. The field of view corresponds to $450 \times 450 \mu \mathrm{m}(\approx 7 \%$ of the simulation cell). (a) Voronoi cells color-coded based on the time-averaged local orientational order parameter $\Psi_{6}(t)$. Colors are specified by the bar at the left. Crosses indicate the positions of pinned particles. (b) Orientational director field, where the complex number $\Psi_{6, i}(t)$ is shown as a $2 \mathrm{D}$ vector, the size of which corresponds to the magnitude of $\Psi_{6, i}(t)$. Outlines of clusters are indicated in black.

time-averaged director $\Psi_{6}$ is defined by

$$
\Psi_{6}(t)=\frac{1}{N} \sum_{i=1}^{N} \Psi_{6, i}(t) .
$$

Orientational clusters are determined via two criteria. First, for a particle to be included in a cluster at time $t$, the time-averaged order parameter has to meet the criterion

$$
\left|\Psi_{6, i}(t)\right| \geqslant 0.5 \text {. }
$$

If particle $i$ is included in a cluster, so may be a neighboring particle $j$, if it meets criterion (6) and the sixfold director has a common orientation. Therefore, we consider the angle $\phi_{i j}$ between the real projection of $\Psi_{6, i}(t), \Psi_{6, j}(t)$ and impose the limit

$$
\cos \left(\phi_{i j}\right)=\frac{\Psi_{6, i} \cdot \Psi_{6, j}}{\left|\Psi_{6, i}\right|\left|\Psi_{6, j}\right|} \geqslant 0.984 .
$$

The application of these two criteria is illustrated in Fig. 1.

\section{CLUSTER ANALYSIS}

Applying the criteria introduced in the previous section to the data recorded in computer simulation and experiment, the formation of clusters is examined within the two-step melting process. Therefore, the reduced transition temperatures $\Gamma_{i}^{-1}$ and $\Gamma_{m}^{-1}$ are inherited from our previous study [37], where the KTHNY melting scenario was confirmed for the system at hand. In the same work, it was shown that there is a broadening of the hexatic phase for increased pinning fractions (see Fig. 2). While $\Gamma_{i}^{-1}$ is hardly affected, $\Gamma_{m}^{-1}$ shifts from $\approx 0.0146$ to $\approx 0.0144$ as the pinning fraction is increased from $0.1 \%$ to $0.5 \%$. These findings are based on an analysis of the spatial and dynamic bond order correlation function. Furthermore, we analyzed the orientational correlation time $\xi_{t}$ and an "effective" Frank's constant $K_{A}$, which is the modulus of torsional stiffness in the presence of pinned particles. Our data indicate that in the presence of disorder, the divergent behavior of Frank's constant is shifted to lower temperatures for higher pinning strengths [37]. Since the divergence of $K_{A}$ coincides 


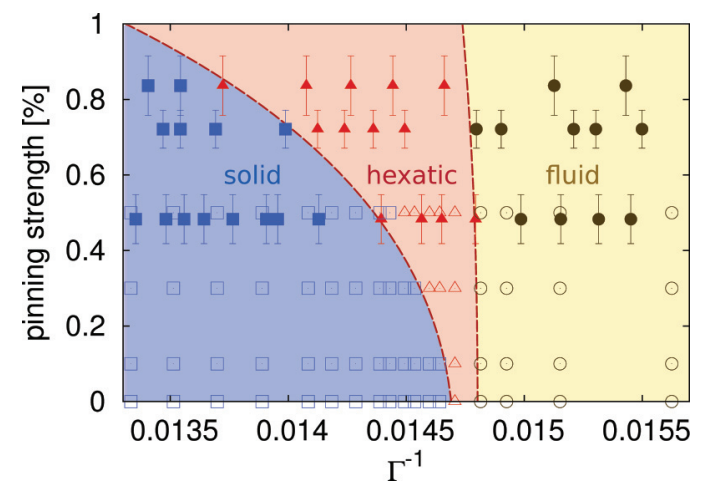

FIG. 2. (Color online) Phase diagram indicating the solid [blue (dark gray)], hexatic fluid [red (medium gray)], and isotropic fluid [yellow (light gray)] phases in the parameter space of the temperature $\propto \Gamma^{-1}$ and pinning strength. Filled symbols represent experimental data, while open symbols correspond to simulation results [37].

with the hexatic $\rightarrow$ solid transition, this implies a strong dependency of $T_{m}$ on disorder, as proposed in Refs. [26][28]. In the following, we revisit the continuous, disorderinduced melting scenario in the context of the development and characteristics of orientational clusters and examine how the latter are influenced by pinning. Figure 3 illustrates the formation of clusters in two exemplary systems with pinning fractions of $0.1 \%$ and $0.5 \%$, respectively. Snapshots are shown at three distinct temperature steps, which, for a pinning fraction of $0.1 \%$, cover all three phases. In the isotropic fluid phase close to $\Gamma_{i}^{-1}$ [Figs. 3(a) and 3(d)], a substantial number of separate clusters can be observed. However, the orientation of these clusters is strongly heterogeneous. In the hexatic phase [Figs. 3(b) and 3(e)], the formerly separated clusters have merged into a large cluster with a homogeneous orientation. Note that, in general, the formation of a uniform cluster does not necessarily collapse with the isotropic $\rightarrow$ hexatic transition point and is highly dependent on the cluster criteria stated in Sec. IV.

As reported in [37], orientational order undergoes spatiotemporal fluctuations throughout the hexatic phase. These critical-like fluctuations occur on time scales beyond the orientational correlation time and are consistent with the continuous nature of the phase transition. In terms of orientational clustering, these fluctuating regions correspond to particles not included in clusters. For further clarification, computer simulation snapshots illustrating the time evolution of clusters on different time scales are shown in Fig. 4. We find the temperature range in which these spatiotemporal patterns occur to depend strongly on the pinning fraction. At $\Gamma^{-1} \approx 0.0146$, the fluctuations have subsided in the system with a pinning fraction of $0.1 \%$ [Fig. 3(c)], while there are still substantial fluctuations in the system with a pinning fraction of $0.5 \%$ [Fig. 3(f)]. Starting from this qualitative observation, we systematically study the co-occurrence of orientational clusters and disordered, fluctuating regions by tracking several quantities related to the formation of clusters versus the effective temperature $\Gamma^{-1}$ for various pinning fractions. First, we measure the abundance of clusters at each specific temperature step. Therefore, the number of clusters is averaged over an observation window of $\approx 10 \tau_{B}$ for the experimental data and $>2000 \tau_{B}$ for the simulation data. In the computer simulation, the result is additionally
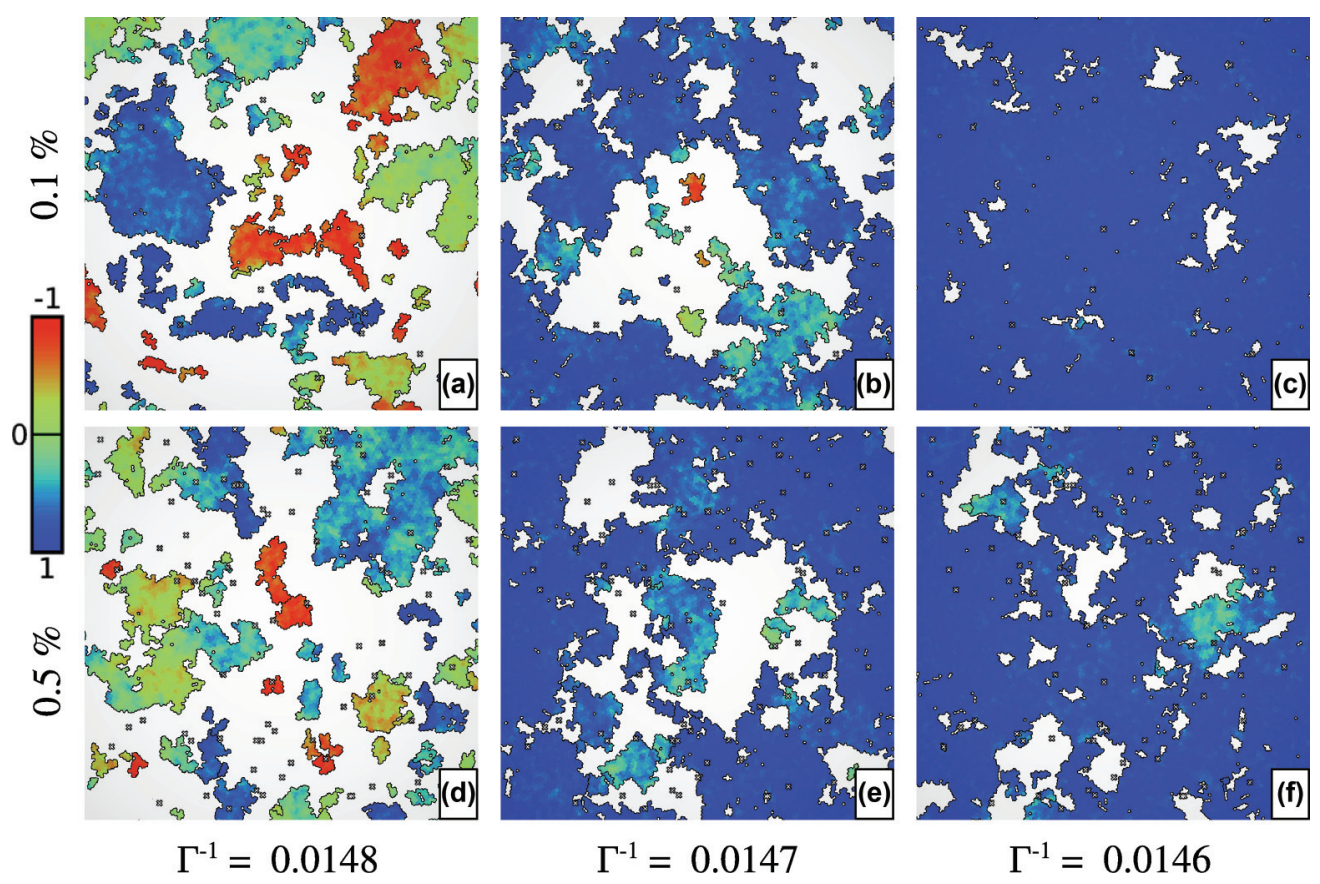

FIG. 3. (Color online) Simulation snapshots of exemplary configurations for pinning fractions $0.1 \%$ (a-c) and $0.5 \%$ (d-f). Voronoi cells are shown for particles included in clusters. The color-code corresponds to the bar at the left, based on the normalized product $\Psi_{6, i} \cdot \Psi_{6} /\left(\left|\Psi_{6, i}\right|\left|\Psi_{6}\right|\right)$. Crosses indicate the positions of pinned particles. At $\Gamma=0.0148$, both systems are in the isotropic fluid phase; the transition to the hexatic phase occurs at $\Gamma=0.0147$. At $\Gamma=0.0146$, the system with less pinning enters the solid phase (c), while the system with a higher pinning fraction remains in the hexatic phase (f) and fluctuations persist. 

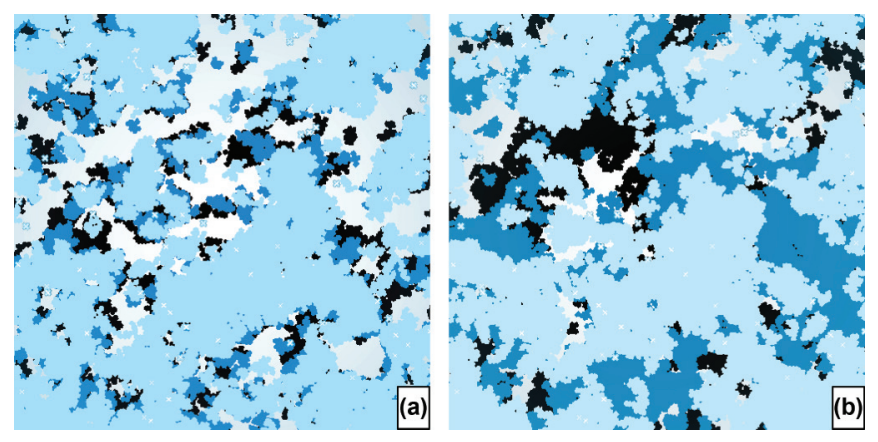

FIG. 4. (Color online) Fluctuating clusters in the hexatic phase ( $\Gamma^{-1}=0.0147,0.5 \%$ pinning). (a) Cluster configurations at three time steps: $t_{0}$ [black], $t_{0}+35 \tau_{B}$ [medium blue (gray)], and $t_{0}+70 \tau_{B}$ [light blue (light gray)]. Voronoi cells are shown for particles included in a cluster. (b) Cluster configurations at three time steps: $t_{1}$ [black], $t_{1}+700 \tau_{B}$ [medium blue (gray)], and $t_{0}+1400 \tau_{B}$ [light blue (light gray)]. Snapshots are from the computer simulation.

averaged over all realizations of disorder for a specific pinning fraction. Our results are shown in Fig. 5; note that in order to account for the different system sizes in experiment and simulation, the cluster number is normalized with respect to the average total particle number $\langle N\rangle$. For both experiment and simulation, we observe that in the fluid phase, the number of clusters $N_{C}$ is high. Close to the isotropic $\rightarrow$ hexatic transition temperature $\Gamma_{i}^{-1} \approx 0.0147$, the number decreases sharply and saturates towards small values close to 1 throughout the hexatic phase. Correspondingly, the normalized number of clusters approaches 0 . This behavior corresponds to the observation of cluster formation in Fig. 3. Additionally, our results capture the influence of pinning disorder on the abundance of clusters: The number of clusters observed at a pinning fraction of $0.5 \%$ systematically exceeds the one observed at $0.1 \%$ pinning over a broad, intermediate temperature interval, which coincides

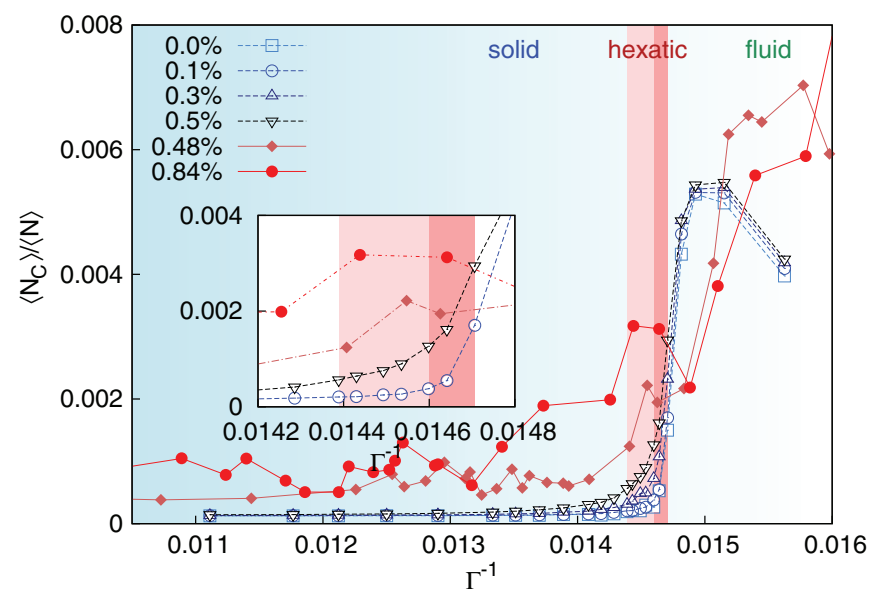

FIG. 5. (Color online) Average of the normalized number of orientational clusters $\left\langle N_{C}\right\rangle /\langle N\rangle$ versus the effective temperature for different pinning strengths. Experimental data are plotted with filled symbols, while open symbols represent numerical data. Lines are guides for the eye. The temperature range of the hexatic phase for $0.1 \%$ pinning is highlighted in red (medium gray); the widening of the hexatic phase at $0.5 \%$ pinning is illustrated in light red (light gray). Inset: Closeup of the average cluster number.

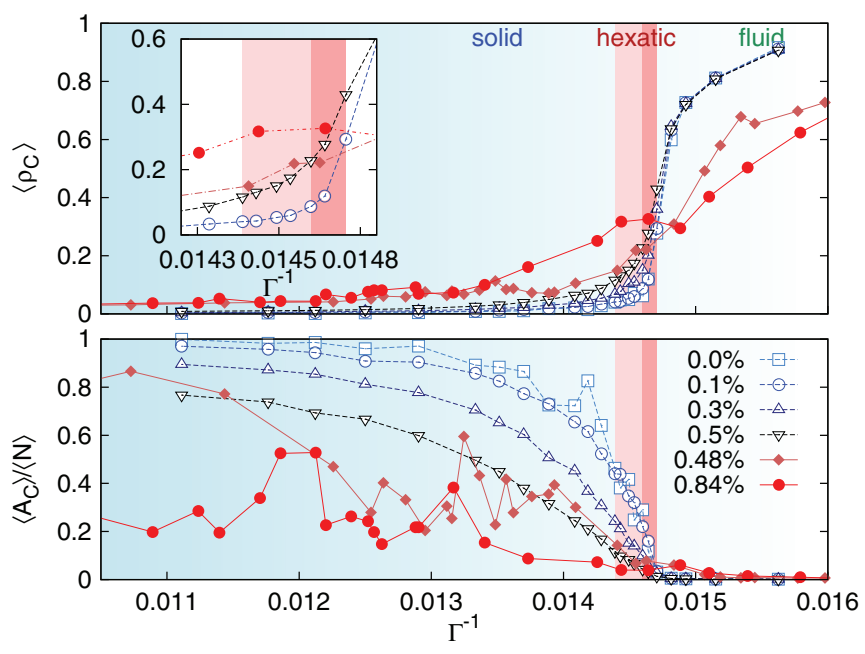

FIG. 6. (Color online) Top: Average ratio $\left\langle\rho_{C}\right\rangle$ of particles outside of clusters versus effective temperature. Inset: Closeup of the behavior in the hexatic phase. Bottom: Average size $\left\langle A_{C}\right\rangle /\langle N\rangle$ of clusters stated as a fraction of the average total particle number. Experimental data are plotted with filled symbols; open symbols represent numerical data. Lines are guides for the eye. As in Fig. 5, the temperature range of the hexatic phase is highlighted in red (gray) for two distinct pinning fractions.

with the widened temperature range of the hexatic phase. This picture is supported by the analysis of fluctuating disordered regions, i.e., regions excluded from clusters. We track the average number density of particles which are excluded from clusters versus the effective temperature, at which the averaging routine depicted above is applied. For every time step, the number of particles excluded from clusters is detected and divided by the total number of particles. The resulting number density $\rho_{C}$ is averaged over all time steps in the observation window. Our results are shown in Fig. 6 (top). We find that the ratio of particles excluded from clusters is close to 1 in the isotropic fluid, drops sharply around $\Gamma_{i}^{-1}$, and approaches 0 at lower temperatures. In agreement with the previous discussion, an increase in the pinning fraction results in a weaker decay of this ratio below $\Gamma_{i}^{-1}$; i.e., a given ratio of excluded particles is maintained over a broader temperature interval than in the case of lower disorder. Again, this temperature interval collapses with the broadened regime of the hexatic phase reported in [37].

As the third quantity, the average size of clusters is shown in Fig. 6 (bottom) for various pinning fractions. The average number of particles included in a single cluster is stated as a fraction of the average total particle number $\langle N\rangle$. This analysis indicates that the formation of clusters spanning the entire system is inhibited by pinning disorder. Instead, the division of the system into a multitude of clusters persists over a broader temperature interval such that, on average, clusters are smaller. Note that for the experimental data, there is less averaging and the average size of clusters fluctuates strongly. However, the qualitative influence of pinning disorder is captured by both experimental and numerical data. The qualitative resemblance of cluster formation in the pure case and in the presence of disorder implies that the emergence of fluctuating orientational clusters is intrinsic to the two-stage melting scenario, not just 

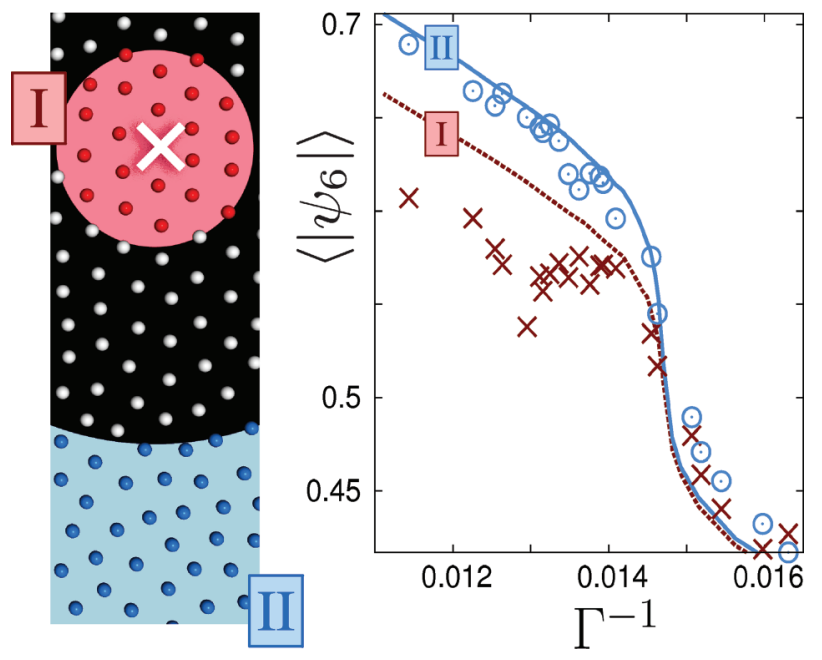

FIG. 7. (Color online) Left: Computer simulation snapshot illustrating the subdivision into regions close to (I) and far from (II) pinned particles. Right: Instantaneous value of the order parameter $\psi_{6}$ in regions I and II versus the effective temperature $\Gamma^{-1}$. Lines correspond to computer simulation data (pinning fraction, $0.5 \%$ ); experimental data are represented by symbols (crosses, region I; circles, region II; pinning fraction, $0.48 \%$ ).

an effect introduced by the presence of pinned particles. As reported in [37], an increase in the pinning fraction induces a shift of $T_{m}$ towards lower values. Here, this behavior is reflected by the inhibited formation of orientational clusters.

For a closer investigation of the impact of pinning disorder on the formation of orientational clusters, we introduce a subdivision of the system into three regions. Region I represents the vicinity of pinned particles and comprises all particles within a radius of $8 d$ around a pinning site (including the pinned particles themself). Region II contains all particles which are more than $24 d$ away from the closest pinning center, thus being presumably unaffected by pinning disorder. Particles contained in neither region I nor region II are neglected for the purpose of the following analysis. The subdivision of the system according to these criteria is illustrated in Fig. 7 (left). We analyze the magnitude of the instantaneous bond orientational order parameter $\psi_{6}$ in regions I and II, respectively. Our results are shown in Fig. 7 (right). A general trend can be observed: On average, orientational order is reduced in the vicinity of pinned particles (region I) compared to distant particles (region II). This effect is especially pronounced in the hexatic and solid phase. No significance can be detected in the isotropic fluid phase, which is expected to be caused by the intrinsic large degree of disorder in an isotropic system.

As a further step, we consider the conditional probability for a particle being part of a cluster, given the fact that the particle belongs to region I or II, respectively (Fig. 8). Thereby, we observe that in the isotropic fluid, particles in region I are in fact slightly more likely to be included in a cluster than particles in region II. Thus, the emergence of small clusters at $T>T_{i}$ occurs preferentially in the vicinity of pinned particles. However, this behavior is inverted at lower temperatures. In the hexatic and solid phase, where single clusters cover large parts of the system, particles in the neighborhood of pinning

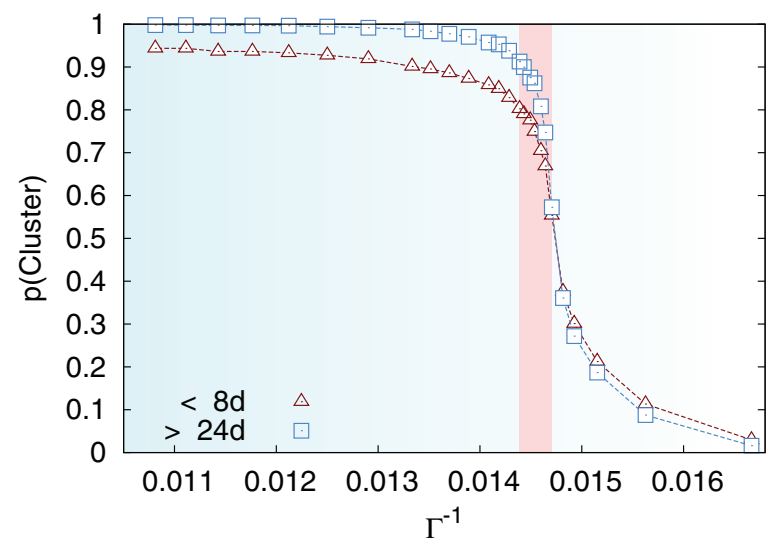

FIG. 8. (Color online) Conditional probability that a particle belongs to a cluster given that it is located in region I (triangles) or region II (squares) versus effective temperature $\Gamma^{-1}$. Data were obtained with computer simulations; the pinning fraction corresponds to $0.5 \%$. Lines are guides for the eye. The temperature range of the hexatic phase is highlighted in light red (gray).

sites are significantly less likely to be part of a cluster. Thus, at low temperatures, pinned particles inhibit the formation of clusters by deteriorating orientational order in their vicinity, while they stabilize orientational order at high temperatures. This result is in good agreement with a previous analysis of the dynamic properties of particles in regions I and II [37]. Thereby, it was found that the long-term dynamics of particles close to pinning sites is inhibited in the fluid phase, while in the solid phase, particles in region I exhibit increased dynamics compared to those in region II. Interestingly, increased or decreased dynamics are correlated to low or high orientational order, respectively.

In order to verify the continuous nature of the phase transition, we calculate the probability distribution $p\left(\Psi_{L}^{2}\right)$ of the squared order parameter $\Psi_{L}^{2}$. We perform a finite-size analysis by considering various subcell sizes, $L=1 / 2,1 / 4$, $1 / 8$, and $1 / 16$, where $L$ states the side length of a subcell as a fraction of the side length of the total system. For a continuous phase transition, we expect the probability distribution to exhibit a single peak for all temperatures and on all length scales. Our results are shown in Fig. 9 and are in agreement with the continuous nature of the melting process. The probability distribution of $p\left(\Psi_{L}^{2}\right)$ is shown for a broader range of temperatures for the fixed subcell size $L=1 / 4$ in Fig. 10.

\section{DEFECT ANALYSIS}

Following the KTHNY theory, the $2 \mathrm{D}$ melting process is driven by the unbinding of topological defects. Specifically, bound dislocation pairs break into isolated dislocations above $T_{m}$, marking the solid $\rightarrow$ hexatic transition. Above $T_{i}$, dislocations dissociate into single disclinations. The existence of isolated dislocations (i.e., pairs of five- and sevenfold defects) leads to quasi-long-range orientational order and is the fingerprint of the hexatic phase. We approach the hexatic phase by tracking the density of defects, in particular, the number of isolated dislocations. Numerically, isolated dislocations are identified as a fivefold and a sevenfold defect particle 
(a) $\Gamma^{-1}=0.0148$

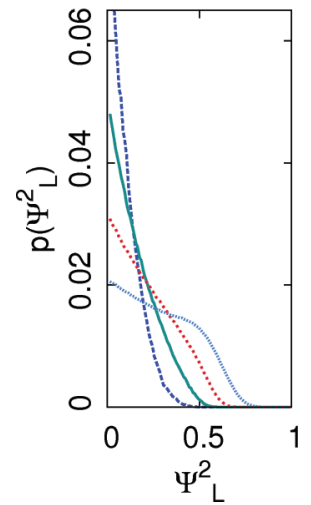

(b) $\Gamma^{-1}=0.0147$

(c) $\Gamma^{-1}=0.0146$
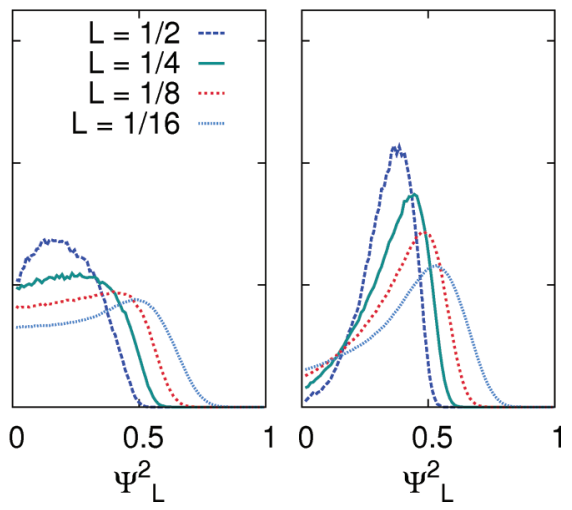

FIG. 9. (Color online) Finite-size analysis of the probability distribution of $\Psi_{L}^{2}$ calculated for subcells of side length $L$ (stated as a fraction of the total box length). Curves are shown for computer simulation data; the pinning fraction corresponds to $0.5 \%$. (a) $\Gamma^{-1}=0.0148$ : isotropic fluid phase, where the peak of the probability distribution is located at $\Psi_{L}^{2}=0$. (b) $\Gamma^{-1}=0.0147$ : fluid $\rightarrow$ hexatic transition, where the peak shifts to intermediate values of $\Psi_{L}^{2}$. (c) $\Gamma^{-1}=0.0146$ : hexatic phase.

which are Voronoi neighbors, and additionally, both have exactly one defect particle in their neighborhood (which is the respective counterpart). Thus, we exclude entangled chains, grain boundaries, and agglomerations of defect particles. However, as discussed in [14], this definition does not exclude close but nonadjacent pairs of dislocations, which, for a sufficiently large Burgers circuit, yield a 0 Burgers vector. Furthermore, the application of periodic boundary conditions affects the counting of defect particles [14]. Since these sources of miscounting affect all our data, a qualitative comparison between defect densities at different pinning fractions is still valid. Additionally, we track the density of bound dislocations, which are as well defined in a way that excludes entanglements
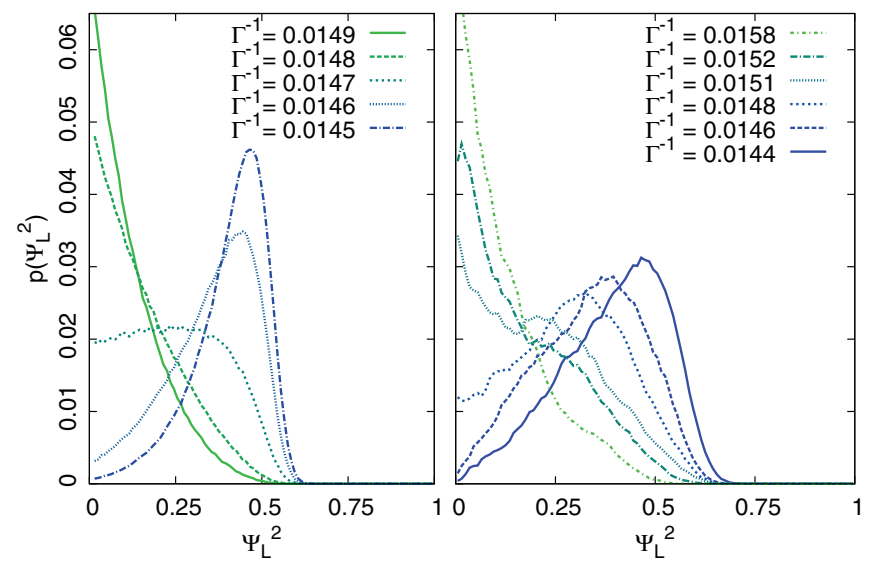

FIG. 10. (Color online) Probability distribution of $\Psi_{L}^{2}$ for simulation (left) and experiment (right) for $L=1 / 4$ (stated as a fraction of the total system dimension). During the crossover from isotropic to hexatic fluid, the location of the peak shifts from $\Psi_{L}^{2}=0$ to intermediate values. The distribution has a single distinct peak, indicating a continuous transition. The pinning fraction is $0.5 \%$ in the simulation and $0.48 \%$ in the experiment.

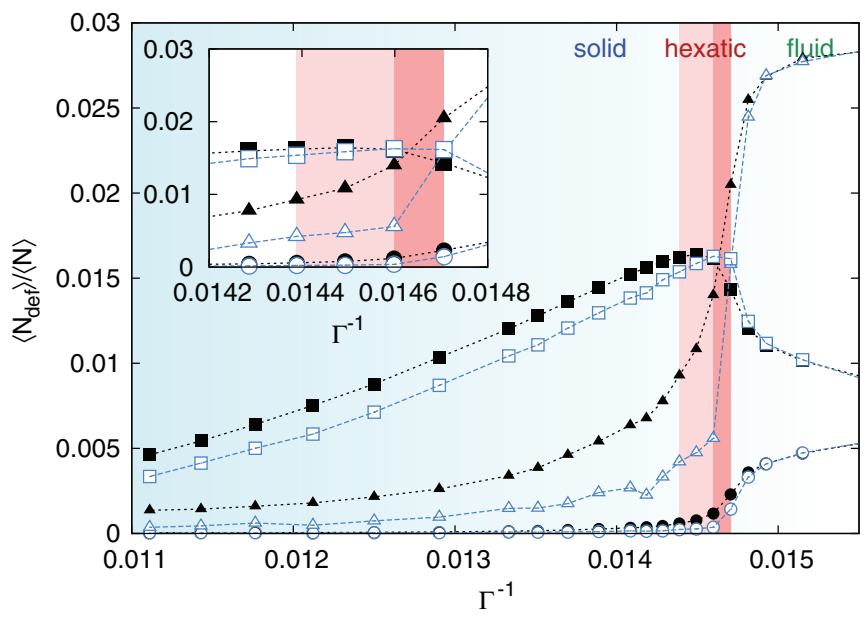

FIG. 11. (Color online) Defect density $\left\langle N_{\text {def }}\right\rangle /\langle N\rangle$ versus effective temperature. The number of particles forming bound dislocations (squares), isolated dislocations (triangles), or single five- or sevenfold disclinations (circles) is stated as a fraction of the total particle number. Data correspond to computer simulations with pinning fraction $0.1 \%$ [open (blue) symbols] and $0.5 \%$ (filled black full symbols). Lines are guides for the eye. The common temperature range of the hexatic phase for both pinning fractions is highlighted in red (medium gray). For $0.5 \%$ pinning, the widened range of the hexatic phase is shown in light red (light gray). Inset: Closeup on the defect density in the (widened) hexatic phase.

and agglomerations of defects. Therefore, we track adjacent pairs of dislocations with no additional defect particles in the Voronoi neighborhood. Finally, single disclinations are tracked, where five- and sevenfold disclinations are treated alike. Figure 11 depicts the recorded defect densities in the computer simulation for two pinning fractions versus the effective temperature. The densities stated are calculated as the total number of particles involved in topological defects of the given kind, $N_{\text {def }}$, divided by the total number of particles $N$. For example, 1 isolated dislocation among 100 particles yields a density of 0.02 , and 1 bound dislocation among 100 particles yields 0.04 . In some analogy to findings reported in [14], we observe that not only single disclinations, but also (isolated) dislocations are abundant in the isotropic fluid phase as well. Below the transition temperature to the hexatic regime, the density of both single disclinations and isolated dislocations decays, while the density of bound dislocations rises, indicating the formation of bound dislocation pairs. We find that increasing the fraction of pinned particles from $0.1 \%$ to $0.5 \%$ leads to a slight systematic increase in all the defect densities, which reflects the fact that pinned particles severly impair translational order. Since, in general, pinned particles are not placed at ideal lattice positions, additional defects have to be introduced in order to accommodate them in a crystal. Furthermore, increasing the fraction of pinned particles has a pronounced effect on the density of isolated dislocations. While for a pinning fraction of $0.1 \%$, the density of isolated dislocations drops sharply at $\Gamma^{-1}=0.0146$, a comparably high density of isolated dislocations is maintained over a broader temperature interval for a pinning fraction of $0.5 \%$. Corresponding to previous findings, this temperature 
range coincides with the widened range of the hexatic phase reported in [37]. Thus, the increased density of isolated dislocations at higher pinning fractions reflects our previous finding of a broadened hexatic phase on the level of topologic defects.

\section{CONCLUSIONS}

We have presented a detailed analysis of the formation and dynamics of orientational clusters and the development of topological defects in the context of the disorder-induced melting scenario in two dimensions. Our results are obtained for superparamagnetic colloids whose repulsion strength can be tuned by an external magnetic field. The colloids are confined at a water-substrate interface where quenched disorder is realized by substrate-induced particle pinning. In addition, corresponding computer simulations of 2D parallel dipoles have been performed. We find that the formation of orientational clusters is intrinsically adapted for the two-stage melting scenario and strongly dependent on the strength of quenched disorder. While the solid is characterized by single cluster formation and large cluster sizes, the cluster density and the number of particles outside of clusters increase sharply in the hexatic phase and saturate in the isotropic fluid. The average cluster size decreases continuously towards the hexatic-isotropic transition and drops to 0 in the isotropic fluid. Throughout the melting process (especially at the solid-hexatic transition), quenched disorder favors the formation of multiple clusters and, equivalently, reduces the average cluster size, but simultaneously the number of particles which do not belong to a cluster increases. This reduces the orientational correlation in space and time due to cluster fluctuations and is directly reflected in the local probability analysis where we detected a reduced cluster formation in the proximity of pinned particles in the solid phase, while clusters are preferentially formed close to pinned particles in the isotropic fluid. In this context, the widening of the hexatic phase due to quenched disorder is directly coupled to the distinct (disorder-dependent) characteristics of orientational cluster formation. The development of topological defects shows a similar effect; especially the creation of isolated dislocations is strongly increased by quenched disorder at the solid-hexatic transition.

Future works should address the effect of long-range quenched potentials, which were originally discussed by Nelson [26], and short-range disorder, e.g., fluctuations of quenched external potentials on the scale of particle diameters [32]. This could be explored via various kinds of external potentials on different length scales, from strong pinning via light fields [41] to weak attractive interactions on precisely structured substrates. Alternatively, density functional theory [42-44] or the phase-field crystal model [45] could be a starting point to describe the hexatic phase [46-48], which could, in principle, be formulated also for quenched disorder $[49,50]$. The behavior of an externally disturbed system under nonequilibrium conditions, e.g., temperature quenches, should be of interest: Concerning the competition between critical fluctuations and first-order characteristics, complex nonequilibrium relaxation dynamics might be induced by quenched disorder. In the absence of disorder, such systems were studied in both one-component [25] and two-component [24] systems and revealed interesting properties. Last, but not least, other types of disorder should be explored systematically, including rough disordered substrates and disorder which is not quenched on the time scale of the measurements.

\section{ACKNOWLEDGMENTS}

We thank T. Neuhaus and C. V. Achim for helpful discussions. This work was supported by the DFG within the SFB TR6 (Projects C2, C,3 and C4) and by the HHU graduate school POROSYS.
[1] J. M. Kosterlitz and D. J. Thouless, J. Phys. C 5, L124 (1972).

[2] J. M. Kosterlitz and D. J. Thouless, J. Phys. C 6, 1181 (1973).

[3] B. I. Halperin and D. R. Nelson, Phys. Rev. Lett. 41, 121 (1978).

[4] D. R. Nelson and B. I. Halperin, Phys. Rev. B 19, 2457 (1979).

[5] A. P. Young, Phys. Rev. B 19, 1855 (1979).

[6] N. D. Mermin, Phys. Rev. 176, 250 (1968).

[7] S. T. Chui, Phys. Rev. B 28, 178 (1983).

[8] M. Glaser and N. Clark, Adv. Chem. Phys. 83, 543 (1993).

[9] K. J. Strandburg, Rev. Mod. Phys. 60, 161 (1988).

[10] Y. Saito, Phys. Rev. Lett. 48, 1114 (1982).

[11] H. Weber, D. Marx, and K. Binder, Phys. Rev. B 51, 14636 (1995).

[12] E. P. Bernard and W. Krauth, Phys. Rev. Lett. 107, 155704 (2011).

[13] A. H. Marcus and S. A. Rice, Phys. Rev. Lett. 77, 2577 (1996).

[14] Y. Han, N. Y. Ha, A. M. Alsayed, and A. G. Yodh, Phys. Rev. E 77, 041406 (2008).

[15] S. Prestipino, F. Saija, and P. V. Giaquinta, Phys. Rev. Lett. 106, 235701 (2011).
[16] Y. Peng, Z. Wang, A. M. Alsayed, A. G. Yodh, and Y. Han, Phys. Rev. Lett. 104, 205703 (2010).

[17] S. Z. Lin, B. Zheng, and S. Trimper, Phys. Rev. E 73, 066106 (2006).

[18] N. Gribova, A. Arnold, T. Schilling, and C. Holm, J. Chem. Phys. 135, 054514 (2011).

[19] R. E. Kusner, J. A. Mann, J. Kerins, and A. J. Dahm, Phys. Rev. Lett. 73, 3113 (1994).

[20] K. Zahn and G. Maret, Phys. Rev. Lett. 85, 3656 (2000).

[21] H. H. von Grünberg, P. Keim, K. Zahn, and G. Maret, Phys. Rev. Lett. 93, 255703 (2004).

[22] P. Keim, G. Maret, and H. H. von Grünberg, Phys. Rev. E 75, 031402 (2007).

[23] P. Dillmann, G. Maret, and P. Keim, J. Phys.: Condens. Matter 20, 404216 (2008).

[24] L. Assoud, F. Ebert, P. Keim, R. Messina, G. Maret, and H. Löwen, Phys. Rev. Lett. 102, 238301 (2009).

[25] P. Dillmann, G. Maret, and P. Keim, Phase transition in two dimensions far from thermal equilibrium, arXiv:1303.6821. 
[26] D. R. Nelson, Phys. Rev. B 27, 2902 (1983).

[27] S. Sachdev and D. R. Nelson, J. Phys. C-Solid State Physics 17 5473 (1984).

[28] M.-C. Cha and H. A. Fertig, Phys. Rev. Lett. 74, 4867 (1995).

[29] S. Herrera-Velarde and H. H. von Grünberg, Soft Matter 5, 391 (2009).

[30] R. E. Kusner, J. A. Mann, and A. J. Dahm, Phys. Rev. B 49, 9190 (1994).

[31] A. Pertsinidis and X. S. Ling, Phys. Rev. Lett. 100, 028303 (2008).

[32] D. Carpentier and P. Le Doussal, Phys. Rev. Lett. 81, 1881 (1998).

[33] P. Yunker, Z. Zhang, and A. G. Yodh, Phys. Rev. Lett. 104, 015701 (2010).

[34] T. Kawasaki and H. Tanaka, J. Phys.: Condens. Matter 23, 194121 (2011).

[35] J. Schockmel, E. Mersch, N. Vandewalle, and G. Lumay, Phys. Rev. E 87, 062201 (2013).

[36] P. Hartmann, A. Douglass, J. C. Reyes, L. S. Matthews, T. W. Hyde, A. Kovacs, and Z. Donko, Phys. Rev. Lett. 105, 115004 (2010).
[37] S. Deutschländer, T. Horn, H. Löwen, G. Maret, and P. Keim, Phys. Rev. Lett. 111, 098301 (2013).

[38] In Ref. [37], this expression was erroneously given without a prefactor $1 / 4 \pi$.

[39] F. Ebert, P. Dillmann, G. Maret, and P. Keim, Rev. Sci. Instrum. 80, 083902 (2009).

[40] K. Binder, Rep. Prog. Phys. 60, 487 (1997).

[41] T. O. E. Skinner, S. K. Schnyder, D. G. A. L. Aarts, J. Horbach, and R. P. A. Dullens, arXiv:1302.2896 (2013).

[42] Y. Singh, Phys. Rep. 207, 351 (1991).

[43] H. Löwen, Phys. Rep. 237, 249 (1994).

[44] S. van Teeffelen, N. Hoffmann, C. N. Likos, and H. Löwen, Europhys. Lett. 75, 583 (2006)

[45] H. Emmerich, H. Löwen, R. Wittkowski, T. Gruhn, G. I. Tóth, G. Tegze, and L. Gránásy, Adv. Phys. 61, 665 (2012).

[46] V. N. Ryzhov and E. E. Tareyeva, Phys. Rev. B 51, 8789 (1995).

[47] V. N. Ryzhov and E. E. Tareyeva, Physica A 314, 396 (2002).

[48] A. Jaiswal, S. L. Singh, and Y. Singh, Phys. Rev. E 87, 012309 (2013).

[49] M. Schmidt, Phys. Rev. E 66, 041108 (2002).

[50] P. P. F. Wessels, M. Schmidt, and H. Löwen, Phys. Rev. Lett. 94, 078303 (2005). 